\title{
Tailoring Conformation-Induced Chromism of Polythiophene Copolymers for Nucleic Acid Assay at Resource Limited Settings
}

\author{
Deepa Rajwar, ${ }^{\dagger, \S}$ Gopal Ammanath, ${ }^{\dagger, \downarrow, \S}$ Jamal Ahmed Cheema, ${ }^{\dagger, \S}$ Alagappan Palaniappan, ${ }^{\dagger, \S}$
} Umit Hakan Yildiz, ${ }^{*}, \|$ and Bo Liedberg $*, \dagger,+, \S$

${ }^{\dagger}$ Center for Biomimetic Sensor Science and ${ }^{\ddagger}$ Nanyang Institute of Technology in Health and Medicine, Interdisciplinary Graduate School, Nanyang Technological University, Singapore 637553

${ }^{\S}$ School of Materials Science and Engineering, Nanyang Technological University, Singapore 639798

"Department of Chemistry, Izmir Institute of Technology, Urla, 35430 Izmir, Turkey

\section{Supporting Information}
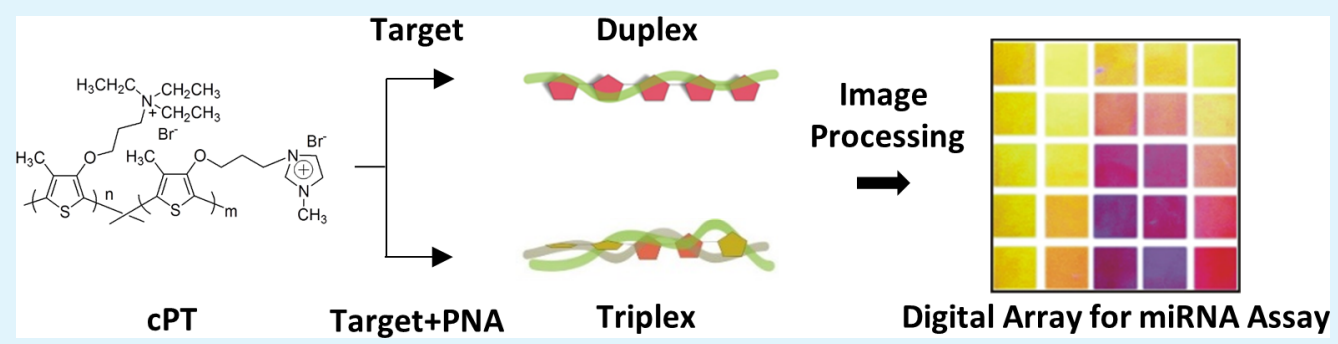

ABSTRACT: Here we report on the design and synthesis of cationic water-soluble thiophene copolymers as reporters for colorimetric detection of microRNA (miRNA) in human plasma. Poly(3-alkoxythiophene) (PT) polyelectrolytes with controlled ratios of pendant groups such as triethylamine/1-methyl imidazole were synthesized for optimizing interaction with target miRNA sequence (Tseq). Incorporation of specific peptide nucleic acid (PNA) sequences with the cationic polythiophenes yielded distinguishable responses upon formation of fluorescent PT-PNA-Tseq triplex and weakly fluorescent PT-Tseq duplex, thereby enabling selective detection of target miRNA. Unlike homopolymers of PT (hPT), experimental results indicate the possibility of utilizing copolymers of PT (cPT) with appropriate ratios of pendant groups for miRNA assay in complex matrices such as plasma. As an illustration, colorimetric responses were obtained for lung cancer associated miRNA sequence (mir21) in human plasma, with a detection limit of $10 \mathrm{nM}$, illustrating the feasibility of proposed methodology for clinical applications without involving sophisticated instrumentation. The described methodology therefore possesses high potential for low-cost nucleic acid assays in resource-limited settings.

KEYWORDS: miRNA, conjugated polyelectrolytes, colorimetric assay, PNA, polythiophene

\section{INTRODUCTION}

Over the past few decades, conjugated polyelectrolytes (CPE) have attracted significant interest in areas such as light-emitting diodes, ${ }^{1}$ solar cells, ${ }^{2}$ field-effect transistors, ${ }^{3}$ and biosensors ${ }^{4-6}$ relying on delocalized $\pi$-electrons along their conjugated backbone that yield unique optical and electronic properties. The optical properties of CPE could be varied with external stimuli such as temperature, ${ }^{7-9}$ solvent or $\mathrm{pH}$ change, ${ }^{10,11}$ electric field, ${ }^{12}$ and interaction with other molecules that may induce chirality in the supramolecular structure, subsequently changing the conjugation length of $\mathrm{CPE} .^{13-17}$ Among biocompatible CPEs, cationic polythiophenes (PT) have been explored for biosensing because of their superior optical and electronic properties. $^{18,19}$ Substituted PTs are of further interest because they are chemically and photochemically stable. As described elsewhere, optical transitions of PT are due to the conformational alternations in backbone that increase or reduce the effective conjugation length leading to red- or blueshift, respectively, of UV-vis absorption maximum. ${ }^{20}$ The extent of conformational alternations in backbone is mainly controlled by energy required for interring twisting, which is less than $2.5 \mathrm{kcal} \mathrm{mol}^{-1}$ for disubstituted thiophene. ${ }^{20}$ The dihedral angle between the conjugated rings was calculated to be $67.8^{\circ}$ for 3,4-dimethyl substituted thiophene, while cyclopentene substitution on to polymer was reported to reduce the steric hindrance thereby lowering twisting angle $\left(55-56^{\circ}\right)$. In this context, addition of pendant groups with varying chemical moieties to the conjugated polymer would further alter the twisting angle between rings. Therefore, we have introduced two different pendant groups in the same conjugated polymer backbone by statistical copolymerization of two monomers for tuning interring twisting of PT upon complexation with miRNA.

Complexation mechanisms of PT with miRNA have been reported elsewhere. ${ }^{21}$ Color transition is a macroscopic response

Received: December 14, 2015

Accepted: March 9, 2016

Published: March 9, 2016 
(A)

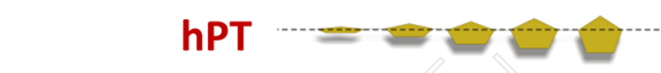

hPT-PNA-Tseq Triplex

hPT or cPT Tseq Duplex

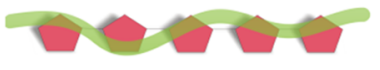

cPT-PNA-Tseq Triplex

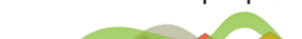

(B)

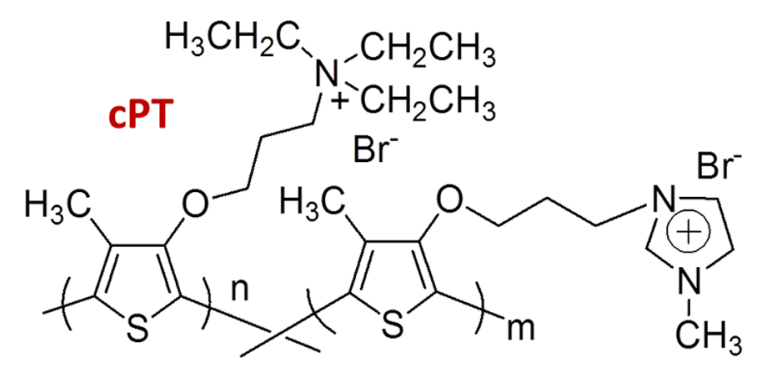

(C)

CPT
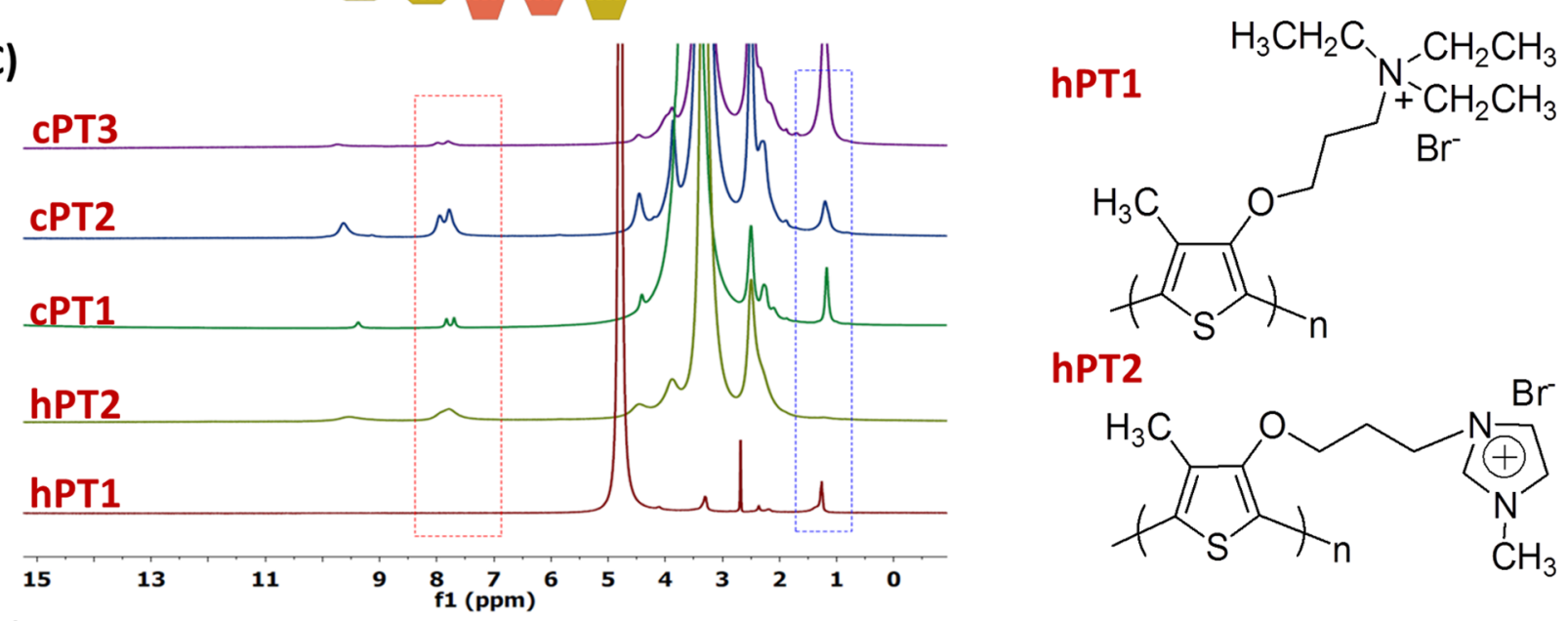

hPT2

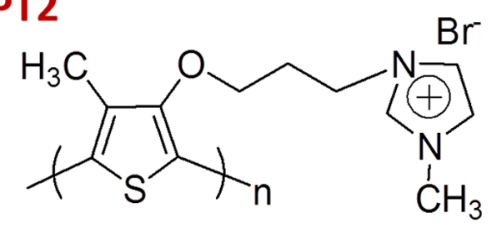

(D)
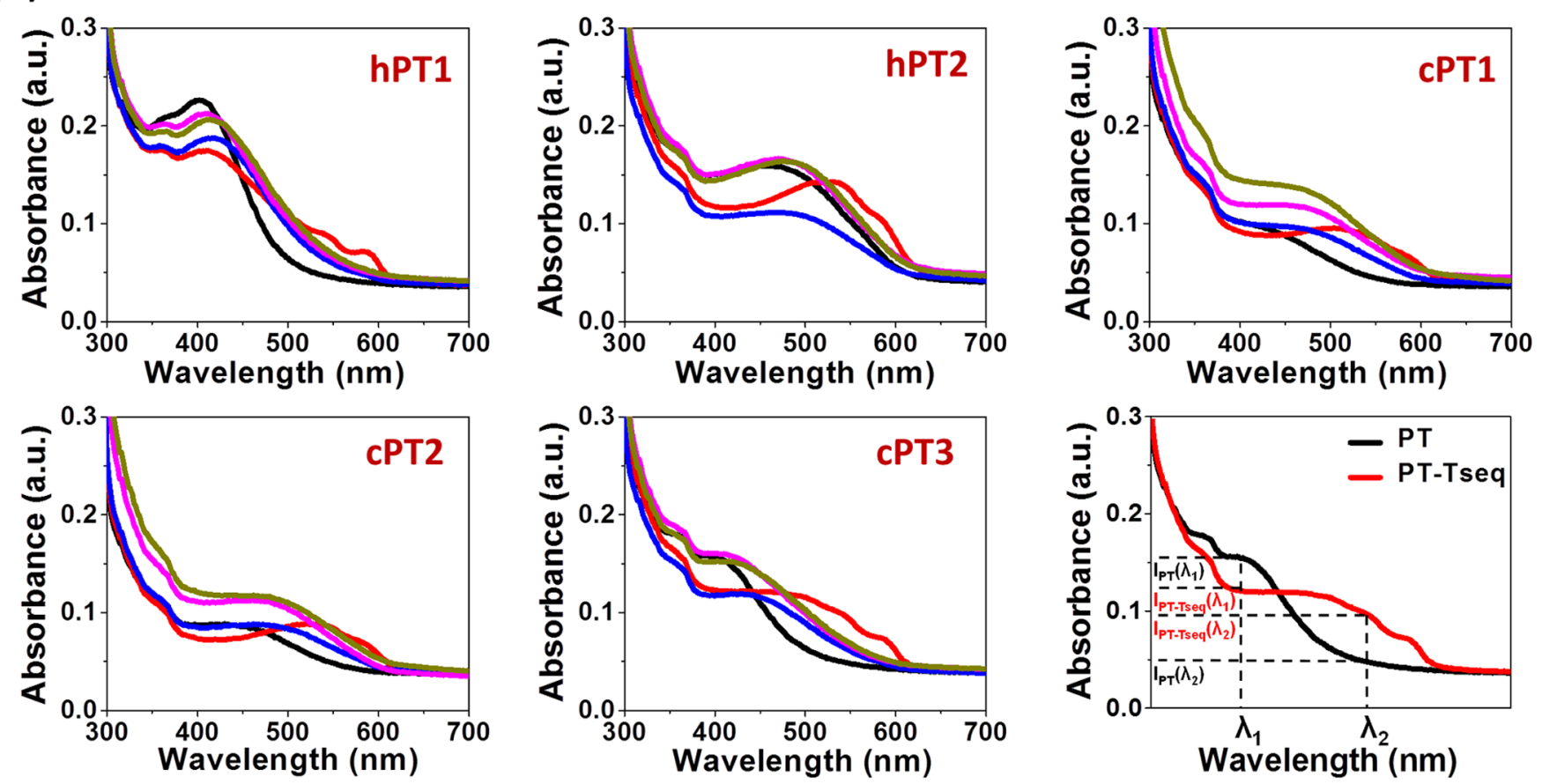

—PT —PT - Tseq —PT - NCseq — PT - PNA - Tseq — PT - PNA - NCseq

Figure 1. (A) Schematic of PT-Tseq/NCseq duplex and PT-PNA-Tseq triplex formation using homo- and copolymers. (B and C) Chemical structures of hPTs and cPT and their corresponding ${ }^{1} \mathrm{H}$ NMR spectra illustrating peaks for hydrogen atoms of imidazole group (red box) and terminal amine's methyl group (blue box). (D) Absorption spectra of PT (150 $\mu \mathrm{M})$ in DI with $1.4 \mu \mathrm{M}$ miRNA and an illustration for calculating the change $(\Delta)$ in absorbance ratio at $\lambda_{1}$ and $\lambda_{2}$.

of PT upon complexation with miRNA as a consequence of twisting-planarization of polymer backbone. Though this unique property of PT has been extensively used for sensing applications, homopolymers of $\mathrm{PT}(\mathrm{hPT})$ are often associated 
with uncontrolled aggregation and interferences from complex matrices that largely limit assay development. Here we propose that by controlling monomer ratios of two thiophene species interring twisting could be tuned to obtain copolymers (cPT) that yield tunable colorimetric response upon interaction with miRNA thereby enabling miRNA assay in human plasma. Five different PTs, two hPTs (hPT1 and hPT2), and three cPTs (cPT1, cPT2 and cPT3) with varying terminal groups were systematically evaluated for miRNA assay. mir21, a miRNA sequence associated with lung cancer, is utilized in this study as the target sequence (Tseq) for evaluating colorimetric response of PT upon incorporation of a peptide nucleic acid sequence (PNA) complementary to mir21. PT exhibited a distinguishable colorimetric response upon formation of PT-PNA-Tseq triplexes compared to that of PT-PNA-NCseq (noncomplementary sequence) mixtures or $\mathrm{PT}-\mathrm{Tseq} / \mathrm{NCseq}$ duplexes (Figure 1A). Significant optical responses were obtained for the synthesized $\mathrm{cPT}$ compared to those of $\mathrm{hPT}$ owing to the synergistic influence of electrostatic and hydrophobic forces of CPT and their pendant groups (Figure 1B) on miRNA. The proposed nucleic acid assay is based on monitoring color variations of PT upon interaction with Tseq/NCseq in the presence and absence of PNA. In the absence of PNA, the resulting color would be due to $\mathrm{PT}-\mathrm{Tseq} / \mathrm{NCseq}$ duplex formation (weakly fluorescent), whereas in the presence of PNA, the output color would be due to either the formation of duplex (PT-NCseq, weakly fluorescent) or formation of triplex (PT-PNA-Tseq, fluorescent) thereby yielding different color signals.

\section{EXPERIMENTAL SECTION}

Materials and Methods. All the reagents and solvents were obtained from Sigma-Aldrich and used without further purification. PTs were synthesized by oxidative polymerization of their monomers; NMR spectra were recorded on a Bruker Advance $400 \mathrm{MHz}$ spectrometer (Figures $1 \mathrm{C}$ and $\mathrm{S} 1-\mathrm{S} 9$ ). mir21 (Tseq) $5^{\prime}$ UAG CUU AUC AGA CUG AUG UUG A 3' and NCseq (control) $5^{\prime}$ AUG CAU GCA UGC AUG CAU GCA A ' 3 were obtained from IDT (Singapore). The PNA probe, N-TCA ACA TCA GTC TGA TAA GCT A-C, was purchased from Panagene (Daejon, Korea). Steady state absorption and emission spectra were obtained by an Infinite M200Pro tecan plate reader and Thermo Scientific Nanodrop 2000c spectrophotometer. Human plasma was purchased from GeneTex, Taiwan, for spiking and subsequent isolation of miRNA using Macherey-Nagel's Nucleospin miRNA extraction kit. Tris buffer was purchased from Bio-Rad Laboratories (Singapore). MES buffer was purchased from Sigma-Aldrich.

Synthesis of Precursor 1 (3-Methoxy-4-methylthiophene). To a dry round-bottom flask, 1-methyl-2-pyrrolidinone $(6.7 \mathrm{~mL}, 69.80 \mathrm{mmol})$ and sodium methoxide/MeOH (25 wt \%; $17.8 \mathrm{~mL}, 78.50 \mathrm{mmol}$ ) were added. After heating this mixture to $110{ }^{\circ} \mathrm{C}$, 3-bromo-4methylthiophene $(5 \mathrm{~g}, 28.20 \mathrm{mmol})$ and $\mathrm{CuBr}(2.47 \mathrm{~g}, 17.20 \mathrm{mmol})$ were added. The round-bottomed flask was then equipped with a water-cooled condenser and refluxed for 1 day at $110^{\circ} \mathrm{C}$ (Scheme 1A). The reaction mixture was cooled to room temperature and a solution of $1 \mathrm{~g}$ of sodium bromide in $40 \mathrm{~mL}$ of DI water was then added and stirred vigorously for $1 \mathrm{~h}$. The mixture was then filtered and extracted with $50 \mathrm{~mL}$ of diethyl ether five times. The combined organic layers were washed with DI water at least three times and dried over $\mathrm{MgSO}_{4}$. Solvent was then removed by rotary evaporator to yield a light-yellow oil. The light yellow oil was further purified with column chromatography to yield 3-methoxy-4-methylthiophene (2.28 g (63\%)). ${ }^{1} \mathrm{H}$ NMR $\left(400 \mathrm{MHz}, \mathrm{CDCl}_{3}, \delta\right.$, ppm, Figure $\left.\mathrm{S} 1\right) 6.84(1 \mathrm{H}, \mathrm{d})$, $6.18(1 \mathrm{H}, \mathrm{d}), 3.84(3 \mathrm{H}, \mathrm{s}), 2.11(3 \mathrm{H}, \mathrm{s})$.

Synthesis of Precursor 2 (3-(3-Bromo)propoxy-4-methylthiophene). A mixture of 3-methoxy-4-methylthiophene (precursor 1)
(0.84 g, $6.60 \mathrm{mmol})$, 3-bromo-1-propanol $(2.00 \mathrm{~g}, 14.4 \mathrm{mmol})$, and $\mathrm{NaHSO}_{4}(123 \mathrm{mg}, 0.900 \mathrm{mmol})$ in $15 \mathrm{~mL}$ of toluene was added to a two-necked, round-bottomed flask. This reaction mixture was heated at $100{ }^{\circ} \mathrm{C}$ for $24 \mathrm{~h}$ under nitrogen $\left(\mathrm{N}_{2}\right)$ atmosphere (Scheme $1 \mathrm{~B}$ ); meanwhile, the produced methanol was distilled off from the reaction flask. The reaction mixture was allowed to cool to room temperature. Toluene was removed by rotary evaporator, and the remaining reaction mixture was extracted five times with diethyl ether, subsequently washed with water, and dried over magnesium sulfate. Diethyl ether was removed by rotary evaporator. The crude product was purified with column chromatography (silica gel, hexane) to give $1.0 \mathrm{~g}(65 \%)$ of precursor 2 as colorless oil. ${ }^{1} \mathrm{H}$ NMR $(400 \mathrm{MHz}$, $\mathrm{CDCl}_{3}, \delta$, ppm, Figure S2) $6.83(1 \mathrm{H}, \mathrm{d}), 6.19(1 \mathrm{H}, \mathrm{d}), 4.08(2 \mathrm{H}, \mathrm{m})$, $3.59(2 \mathrm{H}, \mathrm{m}), 2.34(2 \mathrm{H}, \mathrm{m}), 2.09(3 \mathrm{H}, \mathrm{s})$.

Synthesis of Monomer 1. To a round-bottomed flask were added 3-(3-bromo)propoxy-4-methylthiophene $(0.27 \mathrm{~g}, 1.15 \mathrm{mmol})$, triethylamine $(26.45 \mathrm{mmol}, 3.7 \mathrm{~mL})$, and THF $(30 \mathrm{~mL})$. The mixture was stirred at $72{ }^{\circ} \mathrm{C}$ for 2 days (Scheme 1C). The crude product was washed with THF to yield $0.30 \mathrm{~g}(77 \%)$ of monomer $1 .{ }^{1} \mathrm{H}$ NMR $\left(400 \mathrm{MHz}, \mathrm{DMSO}-d_{6}, \delta\right.$, ppm, Figure S3) $7.09(\mathrm{~d}, 1 \mathrm{H}), 6.53(\mathrm{~d}, 1 \mathrm{H})$, $4.02(\mathrm{t}, 2 \mathrm{H}), 3.29(\mathrm{~m}, 8 \mathrm{H}), 2.08(\mathrm{~m}, 2 \mathrm{H}), 2.03(\mathrm{~s}, 3 \mathrm{H}), 1.20(\mathrm{t}, 9 \mathrm{H})$. ${ }^{13} \mathrm{C}$ NMR (100 MHz, DMSO-d6, $\delta$, ppm) 155.41, 128.56, 121.09, $97.85,66.61,53.60,52.52,21.88,12.92,7.53$

Synthesis of Monomer 2. To a solution of 3-(3-bromo)propoxy-4methylthiophene $(0.27 \mathrm{~g}, 1.150 \mathrm{mmol})$ in $\mathrm{CH}_{3} \mathrm{CN}(35 \mathrm{~mL})$ in a round-bottomed flask was added 1 -methylimidazole $(1 \mathrm{~mL})$. The mixture was stirred for $48 \mathrm{~h}$ at $70{ }^{\circ} \mathrm{C}$ and then evaporated to dryness (Scheme 1D). After evaporation of the solvent, the crude product was washed with warm ethyl acetate and diethyl ether to yield $0.27 \mathrm{~g}$ (80\%) of monomer 2. ${ }^{1} \mathrm{H}$ NMR $\left(400 \mathrm{MHz}, \mathrm{CDCl}_{3}, \delta\right.$, ppm) 10.12 $(\mathrm{s}, 1 \mathrm{H}), 7.51(\mathrm{~s}, 1 \mathrm{H}) 7.46(\mathrm{~s}, 1 \mathrm{H}), 6.74(\mathrm{~d}, 1 \mathrm{H}), 6.51(\mathrm{~d}, 1 \mathrm{H}), 4.51$ $(\mathrm{t}, 2 \mathrm{H}), 3.98(\mathrm{~m}, 5 \mathrm{H}), 2.39(\mathrm{~m}, 2 \mathrm{H}), 1.97(\mathrm{~s}, 3 \mathrm{H}) .{ }^{13} \mathrm{C}$ NMR $\left(100 \mathrm{MHz}, \mathrm{CDCl}_{3}, \delta\right.$, ppm, Figure S4) 155.10, 137.30, 128.61, 123.58, $122.54,120.33,97.27,66.21,47.33,36.81,29.84,12.75$.

Synthesis of Homopolymer 1. A solution of 3-(4-methyl-3'thienyloxy)propyltriethylammonium bromide (monomer 1) $(270 \mathrm{mg}$, $0.910 \mathrm{mmol}$ ) in $5 \mathrm{~mL}$ of dry $\mathrm{CHCl}_{3}$ was added dropwise to a solution of anhydrous $\mathrm{FeCl}_{3}(590 \mathrm{mg}, 3.640 \mathrm{mmol})$ in $25 \mathrm{~mL}$ of dry $\mathrm{CHCl}_{3}$, and stirred for $24 \mathrm{~h}$ at room temperature under $\mathrm{N}_{2}$ atmosphere and subsequently evaporated to dryness (Scheme $1 \mathrm{E}$ ).

Synthesis of Homopolymer 2. Monomer $2(80 \mathrm{mg}, 0.260 \mathrm{mmol})$ in $5 \mathrm{~mL} \mathrm{CHCl} \mathrm{CH}_{3}$ was added dropwise to a solution of $175 \mathrm{mg}$ $(1.080 \mathrm{mmol})$ of anhydrous $\mathrm{FeCl}_{3}$ in $10 \mathrm{~mL}$ of $\mathrm{CHCl}_{3}$ and stirred for $48 \mathrm{~h}$ at room temperature under $\mathrm{N}_{2}$ atmosphere and subsequently evaporated to dryness (Scheme $1 \mathrm{~F})$.

Synthesis of Copolymer 1 (Monomer 1/Monomer $2=1: 1$ Ratio). A mixture of monomer $1(0.063 \mathrm{mmol}, 21 \mathrm{mg})$ and monomer 2 $(0.063 \mathrm{mmol}, 20 \mathrm{mg})$ in $5 \mathrm{~mL}$ of $\mathrm{CHCl}_{3}$ was added dropwise to a solution of anhydrous $\mathrm{FeCl}_{3}$ of $(0.523 \mathrm{mmol}, 85 \mathrm{mg})$ in $10 \mathrm{~mL} \mathrm{CHCl}$ and stirred for $48 \mathrm{~h}$ at room temperature under $\mathrm{N}_{2}$ atmosphere, then evaporated to dryness (Scheme $1 \mathrm{G}$ ).

Synthesis of Copolymer 2 (Monomer 1/Monomer $2=1: 5$ Ratio). A mixture of monomer $1(0.050 \mathrm{mmol}, 16 \mathrm{mg})$ and monomer 2 $(0.250 \mathrm{mmol}, 84 \mathrm{mg})$ in $10 \mathrm{~mL}$ of $\mathrm{CHCl}_{3}$ was added dropwise to a solution of anhydrous $\mathrm{FeCl}_{3}(1.240 \mathrm{mmol}, 201 \mathrm{mg})$ in $20 \mathrm{~mL}$ of $\mathrm{CHCl}_{3}$ and stirred for $48 \mathrm{~h}$ at room temperature under $\mathrm{N}_{2}$ atmosphere, then evaporated to dryness (Scheme 1G).

Synthesis of Copolymer 3 (Monomer 1/Monomer $2=5: 1$ Ratio). A mixture of monomer $1(0.253 \mathrm{mmol}, 81 \mathrm{mg})$ and monomer 2 $(0.051 \mathrm{mmol}, 17 \mathrm{mg})$ in $10 \mathrm{~mL}$ of $\mathrm{CHCl}_{3}$ was added dropwise to a solution of anhydrous $\mathrm{FeCl}_{3}(1.250 \mathrm{mmol}, 203 \mathrm{mg})$ in $20 \mathrm{~mL}$ of $\mathrm{CHCl}_{3}$ and stirred for $48 \mathrm{~h}$ at room temperature under $\mathrm{N}_{2}$ atmosphere, then evaporated to dryness (Scheme $1 \mathrm{G}$ ). The crude products in synthesis of all homopolymers and copolymers were washed with $\mathrm{CHCl}_{3}$ and diethyl ether to get rid of $\mathrm{FeCl}_{3}$ and starting materials. The collected polymers were dried under vacuum for $24 \mathrm{~h}$.

Colorimetric Analysis. To create a digital array for colorimetric analysis, the samples are placed on UV illumination zone (portable UV lamp, at $365 \mathrm{~nm}, 8 \mathrm{~W}$ ) with white background, and digital images are captured using a camera positioned at a fixed distance from the vials. 
Scheme 1. Synthesis of Compounds of Interest ${ }^{a}$

(A)<smiles>Cc1cscc1Br</smiles><smiles>COc1cscc1C</smiles>

(B)

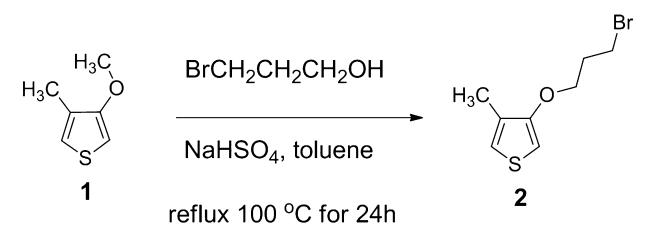

(C)

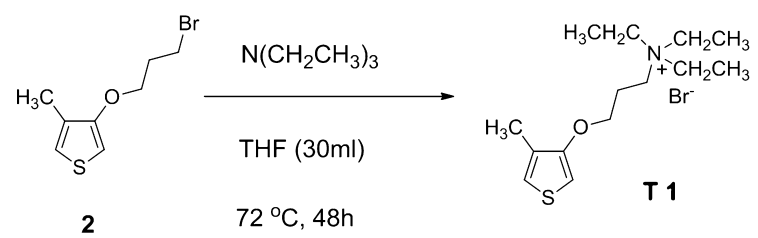

(D)

(E)
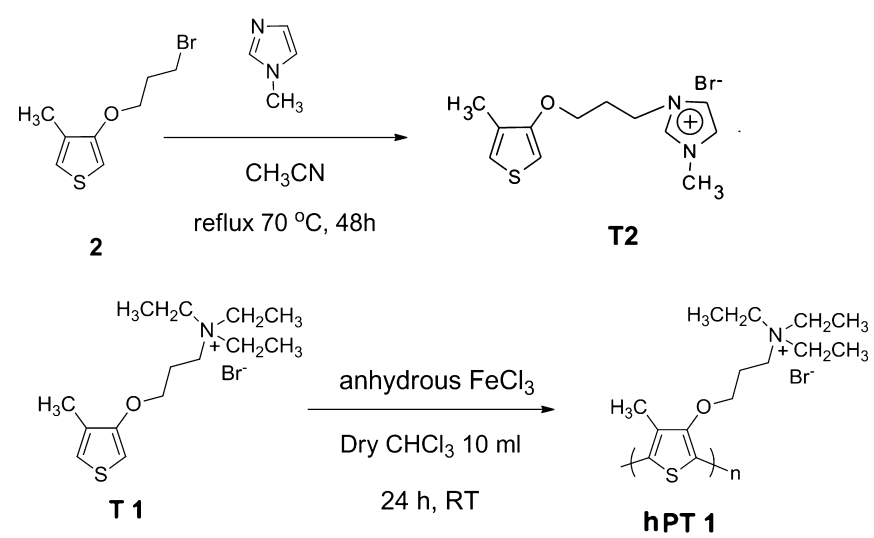

(F)<smiles>Cc1cscc1OCCC[n+]1ccn(C)c1</smiles>

T2

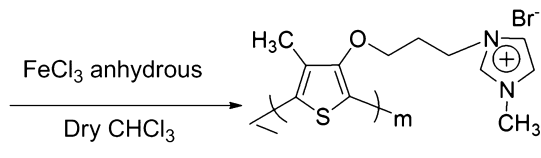

$48 \mathrm{~h}, \mathrm{RT}$

hPT 2

(G)

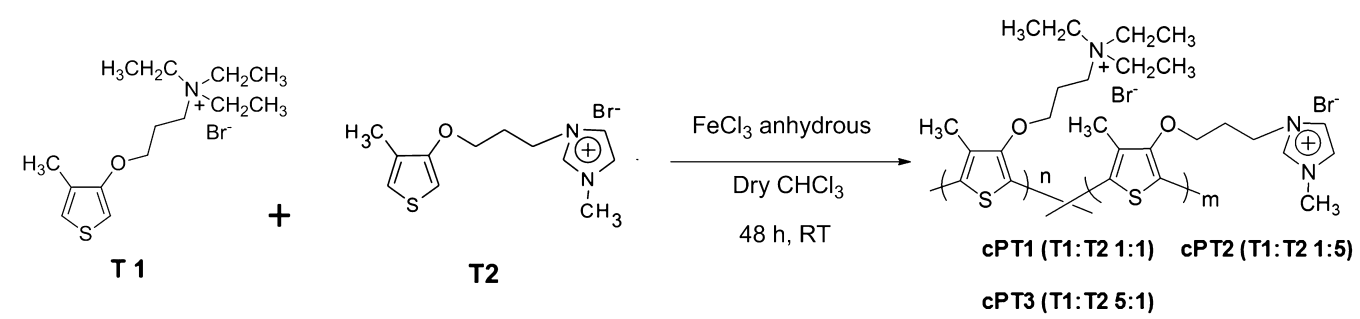

${ }^{a}$ (A) 3-Methoxy-4-methylthiophene; (B) 3-(3-bromo)propoxy-4-methylthiophene; (C) monomer 1 (T1); (D) monomer 2 (T2); (E) hPT1 via oxidative polymerization of $\mathrm{T} 1$; $(\mathrm{F}) \mathrm{hPT} 2$ via oxidative polymerization of $\mathrm{T} 2$; and $(\mathrm{G})$ cPT via oxidative polymerization of $\mathrm{T} 1$ and $\mathrm{T} 2$ in varying ratios.

Consequently, the digital images are transferred to the computer as jpeg files for processing. The raw images are first imported into Adobe Photoshop CS5 software, background color is normalized, and then the centroids of vials $(25 \times 25$ pixel $)$ are utilized to create a digital array. RGB analysis was further carried out using ImageJ image processing software in order to ascertain colorimetric responses.

\section{RESULTS AND DISCUSSION}

Interaction of PT with miRNA. The proton NMR spectra of all PTs in Figure 1C show the trimethylamine and 1-methyl imidazole functional groups for monomer 1 (T1) and monomer 2 (T2), respectively. The $\mathrm{T} 1 / \mathrm{T} 2$ ratio was evaluated from the ${ }^{1} \mathrm{H}$ NMR integral of the characteristic hydrogen 
atoms. For $\mathrm{CPT} 1$, the integral of peaks at $\sim 7.7$ and $7.83 \mathrm{ppm}$ $(\sim 3.3 \mathrm{H}$, indicated by red box $)$ and at $1.17 \mathrm{ppm}(\sim 15.75 \mathrm{H}$, indicated by blue box) corresponding to the hydrogen atoms of imidazole and terminal amine's methyl group, respectively, yield a T1/T2 ratio of 1:0.94 [(15.75/9):(3.3/2)]. The T1/T2 ratios for $\mathrm{CPT} 2$ and $\mathrm{CPT} 3$ were calculated to be $1: 5.03$ and 7.29:1, respectively, indicating successful synthesis of $\mathrm{cPT}$ with varying intermediate ratios of monomers compared to hPT (NMR spectra and ratio calculations are provided in the Figures S5-S9). Further characterization was performed by evaluating the PT spectral characteristics in different buffers, salt concentrations, temperature, and $\mathrm{pH}$ values. No significant changes in PT absorption and emission properties (Figure S10) were observed for changes in buffer conditions.

All the synthesized PTs were subsequently evaluated for their complexation capabilities with miRNA. The changes in the $\pi-\pi^{*}$ transitions that arise from the conformational alternations in the PT backbone upon interaction with miRNA were monitored by absorption and fluorescence spectroscopy. Spectral characteristics recorded using $150 \mu \mathrm{M}$ PT show that the absorbance $\left(I_{\mathrm{PT}}\right)$ maxima of hPT $1, \mathrm{hPT} 2, \mathrm{CPT} 1, \mathrm{cPT} 2$, and cPT3 in DI appear at wavelengths $\left(\lambda_{1}\right) 403,459,415,436$, and $406 \mathrm{~nm}$, respectively, and are associated with random coil conformation of the PT derivatives. ${ }^{14}$ Upon adding $1.4 \mu \mathrm{M}$ of Tseq, the absorbance maximum red-shifted with an observable color transition for all PTs (Figure 1D). To facilitate comparison of colorimetric responses between PTs, the change $(\Delta)$ in absorbance ratio at $\lambda_{1}$ and $\lambda_{2}$ (wavelength corresponding to absorbance peak of PT-Tseq duplex) was evaluated (illustration for cPT3 is shown in Figure 1D). ${ }^{22-24} \Delta_{\text {duplex }}$ $\mathrm{I}\left[I_{\text {duplex }}\left(\lambda_{1}\right) / I_{\text {duplex }}\left(\lambda_{2}\right)-I_{\mathrm{PT}}\left(\lambda_{1}\right) / I_{\mathrm{PT}}\left(\lambda_{2}\right)\right] \mathrm{I}$, and $\Delta_{\text {triplex }}$, I $\left[I_{\text {triplex }}\left(\lambda_{1}\right) / I_{\text {triplex }}\left(\lambda_{2}\right)-I_{\text {duplex }}\left(\lambda_{1}\right) / I_{\text {duplex }}\left(\lambda_{2}\right)\right] \mid$ were observed in the following order; $\Delta_{\mathrm{hPT} 1}(3)>\Delta_{\mathrm{cPT} 3}(1.9)>\Delta_{\mathrm{cPT} 1} \sim \Delta_{\mathrm{cPT} 2}$ $(0.9)>\Delta_{\mathrm{hPT} 2}(0.5)$ and $\Delta_{\mathrm{cPT} 3} \sim \Delta_{\mathrm{hPT} 1}(1)>\Delta_{\mathrm{cPT} 2}(0.6)>$ $\Delta_{\mathrm{hPT} 2}(0.5)>\Delta_{\mathrm{cPT} 1}(0.3)$, respectively. Although hPT1 shows the largest interaction with Tseq, recovery upon PNA addition was relatively lower for hPT1 $\left(\Delta_{\text {triplex }} / \Delta_{\text {duplex }} \sim 33 \%\right)$ when compared to cPT3 ( 53\%).

Figure 1D illustrates that absorbance maxima of PT-Tseq duplex (red spectra) were centered at around $550 \mathrm{~nm}\left(\lambda_{2}\right)$. The deconvolution of these duplex spectra upon baseline correction reveal four minor components with absorbance maximum ranging from 424 to $587 \mathrm{~nm}$ (Figure S11). Absorbance maximum for all the spectra were observed at $\sim 547 \mathrm{~nm}$, which is attributed to the duplex conformation. The remaining minor components, for instance, for hPT1 at 424, 502, and $587 \mathrm{~nm}$, correspond to twisted or planarized conformations. ${ }^{14}$ The most significant spectral shift from 412 to $547 \mathrm{~nm}$ was observed for hPT 1 . The extent of shifts was observed to be in the following order: hPT1 $[135 \mathrm{~nm}]>\mathrm{cPT} 3[133 \mathrm{~nm}]>\mathrm{cPT} 1[106 \mathrm{~nm}]>$ cPT2 $[92 \mathrm{~nm}]>\mathrm{hPT} 2[61 \mathrm{~nm}]$, in agreement with our analysis using absorbance intensity ratios at $\lambda_{1}$ and $\lambda_{2}$ (see above).

Experimental results therefore indicate the possibility of utilizing $\mathrm{CPT}, \mathrm{cPT} 3$ for instance, that possess both favorable quenching and recovery capabilities among all synthesized PT for nucleic acid assay. The main reason for the improvement of cPT behavior is attributed to the introduction of hydrophobic terminal groups (T2) that weakens the electrostatic interaction of $\mathrm{cPT}$ with miRNA. The imidazolium group of $\mathrm{T} 2$ in $\mathrm{cPT}$ would still facilitate cPT-miRNA complexation via both electrostatic and hydrophobic interactions but to a lesser extent as compared to the amine group of $\mathrm{T} 1$, thereby enabling better recovery compared to hPT $1 .^{25,26}$ Experimental results suggest not only that "planarization of backbone" influences optical properties of PT but also that synergistic electrostatic and hydrophobic forces govern the PT-miRNA supramolecular complexation.

Color-Based Sensing Methodology. Spectral characterization (Figures 1D and S12-14) confirms that CPT maximizes the translation of hybridization events into colorimetric responses. Efforts were then devoted to develop a colorimetric assay utilizing $\mathrm{cPT}$. Figure 2A illustrates the proposed strategy for nucleic acid assay that could be carried out using an image processing software. Color normalization was initially performed to minimize interferences due to scattered stray radiation from white background caused by UV lamp. Normalization has been performed by analyzing images as three-component RGB matrices. The initial RGB values of particular image were retrieved by software (imageJ or Photoshop) and normalized according to the following $3 \times 3$ matrix:

$$
\left[\begin{array}{l}
R \\
G \\
B
\end{array}\right]=\left[\begin{array}{ccc}
\frac{255}{R_{w}^{\prime}} & 0 & 0 \\
0 & \frac{255}{G_{w}^{\prime}} & 0 \\
0 & 0 & \frac{255}{B_{w}^{\prime}}
\end{array}\right]\left[\begin{array}{l}
R^{\prime} \\
G^{\prime} \\
B^{\prime}
\end{array}\right]
$$

where $\left(R_{\mathrm{w}}^{\prime}, G_{\mathrm{w}}^{\prime}, B_{\mathrm{w}}^{\prime}\right),\left(R^{\prime}, G^{\prime}, B^{\prime}\right)$, and $(R, G, B)$ are intensities of red, green, and blue components of images of background and before and after normalization, respectively. By applying the described transformation, colors were pronounced while eliminating background interferences. The digital array is subsequently obtained by combining the centroids of all PTs with and without target/PNA. As illustrated in Figure 2A, the first column of each array is the PT reference, which ideally should resemble the second/target column representing the color of mixture of PT-PNA-Tseq. The third to fifth columns are controls, illustrating the color of PT-PNANCseq mixtures, PT-Tseq and PT-NCseq duplexes, respectively.

As an illustration, ideal colorimetric response for Tseq would yield identical color (the color of PT, compare columns one and two in Figure 2B), provided complete triplex formation and weakening of electrostatic interaction between Tseq and PT, whereas column three would yield a color (the color of duplex, dependent on the strength of interaction between PT and Tseq) that is significantly different from columns one and two. Similarly, ideal colorimetric response for NCseq would yield identical colors (color of duplex) for columns four and five. However, one cannot fully exclude partial hybridization/interaction between PNA and NCseq (column five), leading to a colorimetric response that is different from column four.

As observed in Figure 2B, hPT1 and cPT3 exhibited essentially identical colors for columns one and two, whereas cPT3 displayed the most distinguishable color pattern and contrast variations for columns three to five with respect to columns one and two. RGB analysis was subsequently performed for PT-PNA-Tseq triplex and PT-Tseq duplex (Figure 2C). The error bars indicate that the red, green, and blue components variations were less than $10 \%$ for $n=5$. As observed from Figure 2C, cPT3 exhibits prominent transitions in RGB intensities compared to those of other PTs (Figure S15), indicating substantial color transitions upon triplex formation. 
(A)

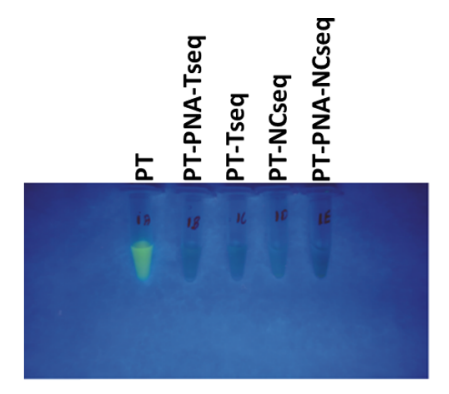

RGB Color Code Analysis

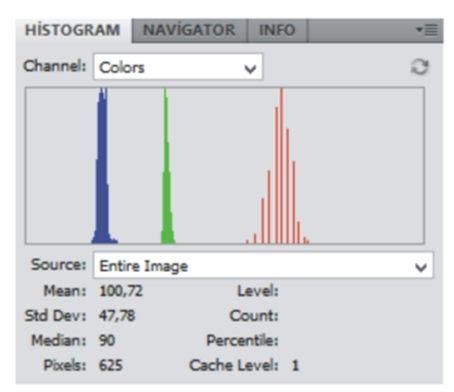

Background

Color

Normalization

\& Pixel Picking

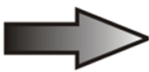

RGB Analysis

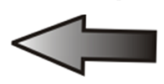

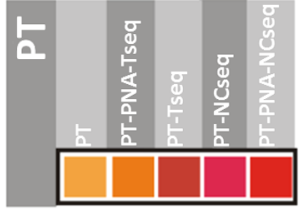

Digital Array

(B)
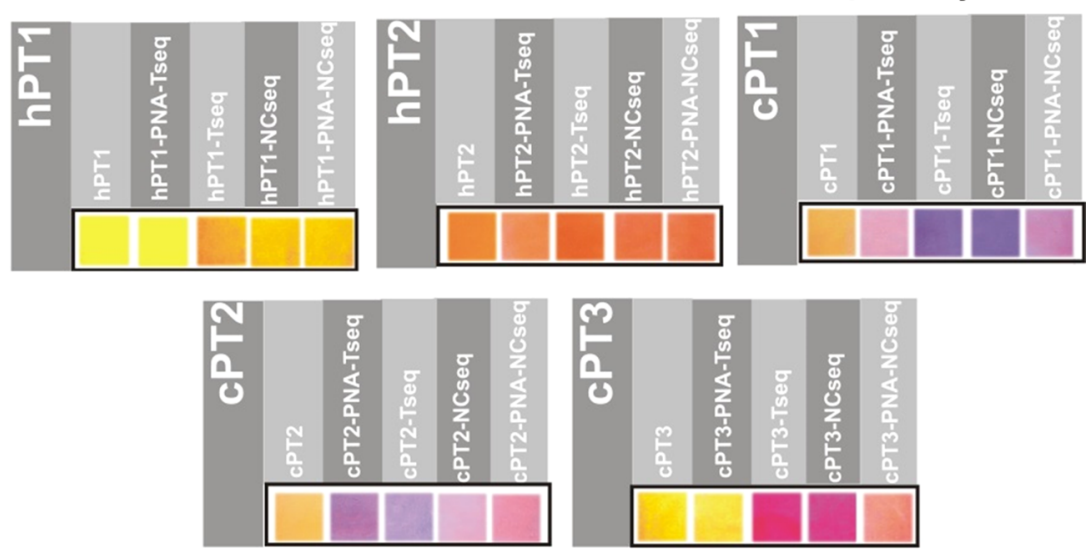

(C)
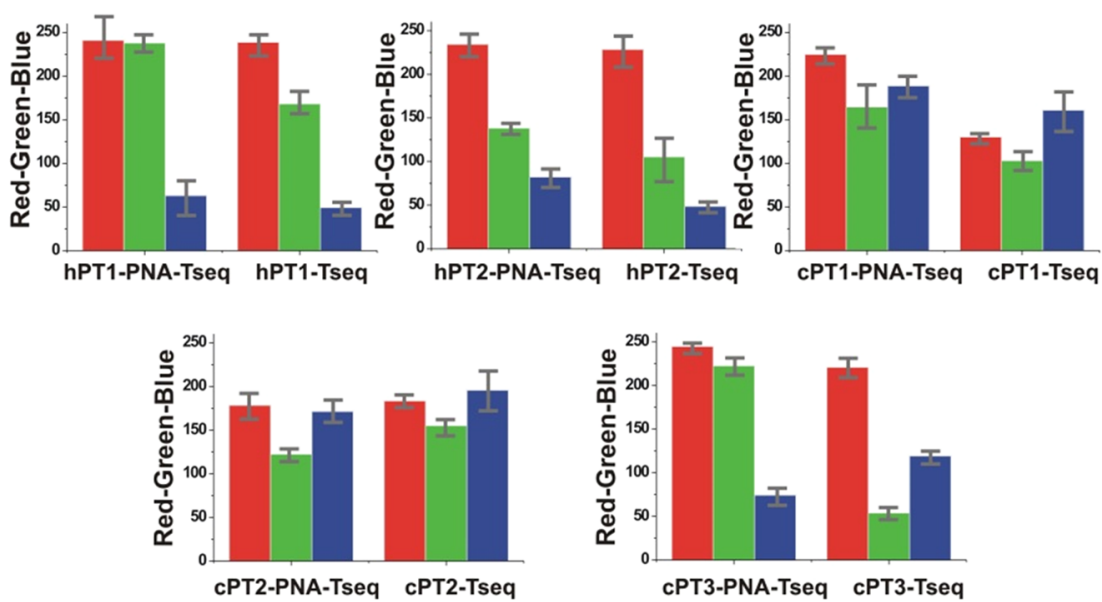

Figure 2. (A) Process of creating color-coded digital array for colorimetric and RGB analysis. (B) Color-coded digital array of all PT for $2 \mu \mathrm{M}$ of Tseq and NCseq in DI, showing colorimetric response for PT (column 1), PT-PNA-Tseq triplex (column 2), PT-Tseq duplex (column 3), PT-NCseq duplex (column 4), and PT-PNA-NCseq mixture (column 5). (C) Differences in RGB intensities for PT-PNA-Tseq triplex and PT-Tseq duplex $(n=5)$ for all digital arrays in B.

The obtained colorimetric responses also concur with the spectral characterization (Figure 1D). As explained previously, spatial arrangement and functional groups at terminal positions could enhance the interaction of cPT3 with miRNA via synergistic backbone planarization and electrostatic/hydrophobic forces. Spectral characterization, digital array responses, and 
(A)
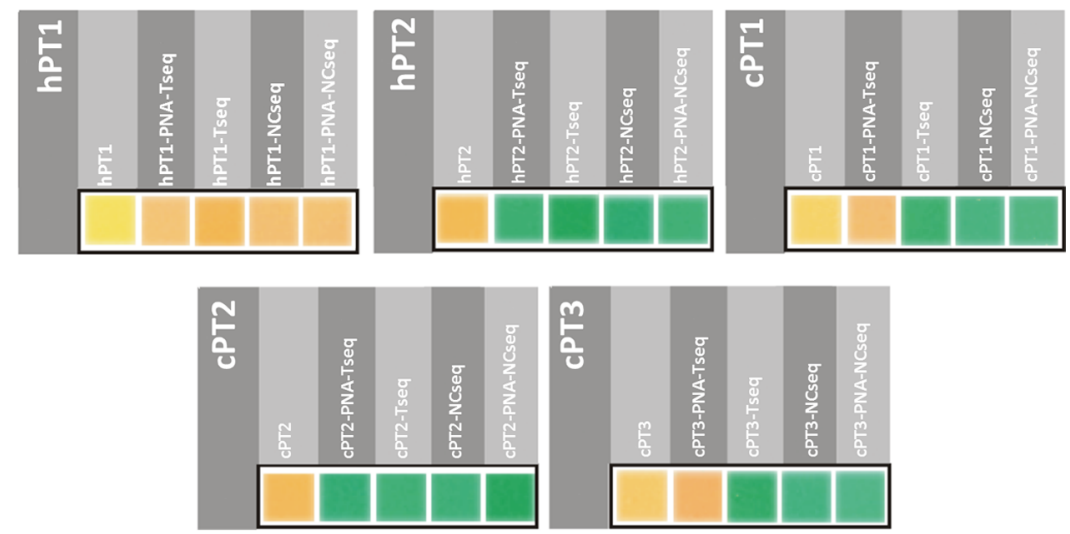

(B)
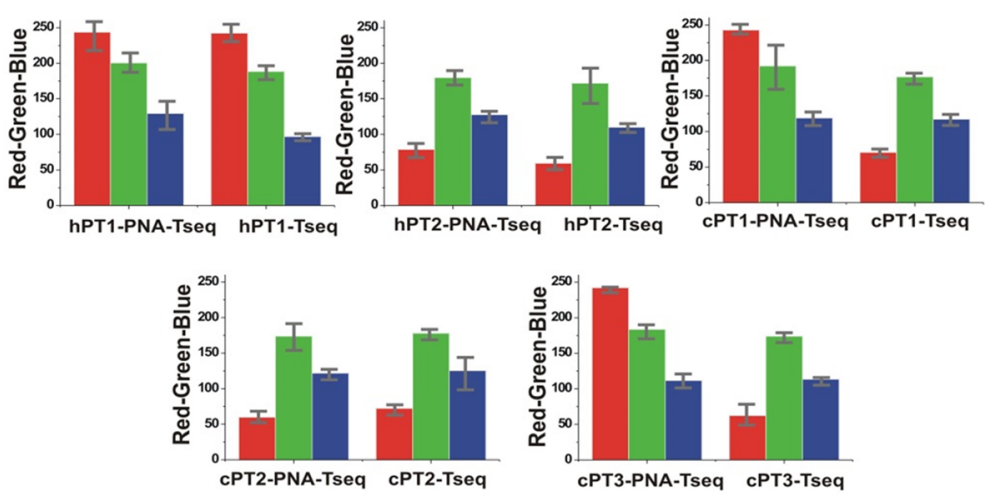

(C)

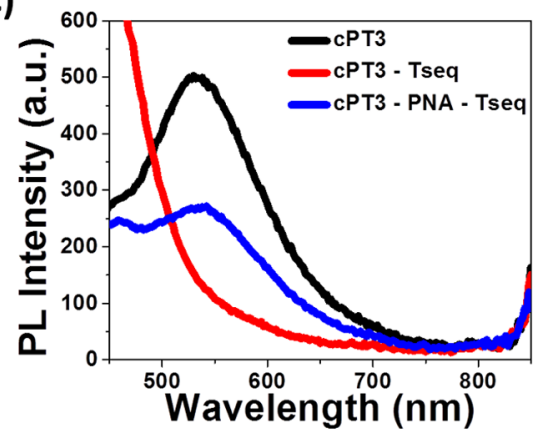

(D)

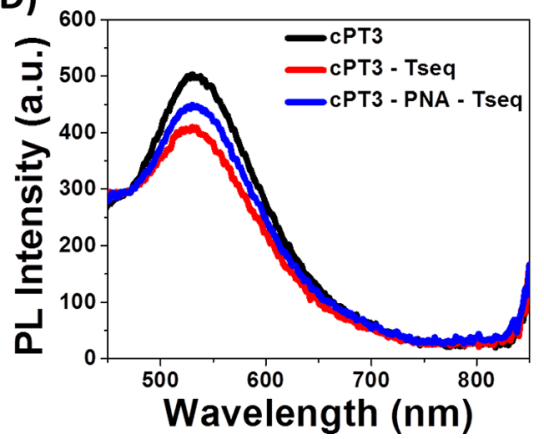

Figure 3. (A) Color-coded digital array of all PT for $3 \mu \mathrm{M}$ of Tseq and NCseq in plasma, showing colorimetric response for PT (column 1), PT-PNA-Tseq triplex (column 2), PT-Tseq duplex (column 3), PT-NCseq duplex (column 4), and PT-PNA-NCseq mixture (column 5). (B) Differences in RGB intensities for PT-PNA-Tseq triplex and PT-Tseq duplex $(n=5)$ for all digital arrays in A. (C and D) PL intensity for 100 and $10 \mathrm{nM}$ Tseq, respectively, in plasma using $10 \mu \mathrm{M}$ cPT3.

RGB analysis suggest that cPT3 could be deployed for miRNA assay in more complex fluids.

Detection of mir21 in Plasma. Colorimetric responses for all PTs were then evaluated with miRNA spiked human plasma samples. As shown in Figure 3A, colorimetric responses were used to obtain the color-coded array using $3 \mu \mathrm{M}$ Tseq and NCseq. In agreement with previous observation using DI, cPT3 exhibited significant colorimetric response. In addition to cPT3, cPT1 also appears to yield distinguishable color development in contrast to its weak response in DI. The improved colorimetric response could be attributed to the higher ionic strength of plasma that might solubilize $\mathrm{cPT} 1$ thereby facilitating miRNA capture. RGB and Delta-E $(\Delta E)$ analysis (Figures 3B and S15) further ascertain superior colorimetric responses for $\mathrm{CPT} 3$ $(\Delta E=64.6)$ and $\mathrm{cPT} 1(\Delta E=60.2)$ compared to that of other PTs $(\Delta E<13)$, indicating their potential for miRNA assay. As shown in Figure S16, naked eye interpretation of colorimetric responses was possible up to $\sim 740 \mathrm{nM}$. It should be noted that at lower miRNA concentrations the color transitions were not easily discernible by naked eye. However, fluorescence recovery analysis of CPT3 illustrates that the proposed methodology enabled detection of Tseq at clinically relevant concentration ranges, as low as $10 \mathrm{nM}$ (Figure 3C,D), by optimizing the stoichiometric ratio of cPT3 $(10 \mu \mathrm{M})$. For instance, at $10 \mu \mathrm{M}$ cPT3, almost complete fluorescence quenching ( $\sim 80 \%)$ was observed with the addition of $100 \mathrm{nM}$ Tseq followed by a substantial fluorescence recovery (35\%) with the addition of complementary PNA, indicating successful detection of Tseq (Figure 3C). Similarly, observable quenching and recovery of fluorescence upon addition of $10 \mathrm{nM}$ Tseq and PNA, respectively, confirms detection of Tseq at clinically relevant concentrations (Figure 3D).

Comparison of Sensing Performance with CPE-Based Nucleic Acid Assays. CPE-based nucleic acid assays have been widely investigated as an alternative to conventional PCR 
assay. For instance, CPE-based competitive detection methodology utilizing short DNA and miRNA sequences competing for hybridization with capture DNA probes have been reported to yield a limit of detection (LOD) of $\sim 1 \mathrm{nM} .^{27}$ Other reports monitoring conformational and colorimetric transitions of a CPE upon interaction with miRNA have been reported to achieve nanomolar level miRNA detection sensitivities. ${ }^{22}$ Recently, polyfluorene-alt-phylene type CPE were utilized to detect DNA in serum samples. ${ }^{28}$ Another FRET-based strategy utilizing cationic poly(phenylenevinylene) and malachite green (as intercalating dye) was found to be sensitive between 10 and $1000 \mathrm{nM}$; however, assay did not provide naked eye detection. ${ }^{29}$ Naked eye detection of nucleic acids by using mixtures of CPEs and gold nanoparticles were successfully presented pending validation with clinical samples. ${ }^{30}$ Electrochemical approaches on quantitative analysis of miRNA have claimed subnanomolar detection sensitivities. ${ }^{31}$ Although detection at nanomolar concentration levels have been reported, most of the reported methodologies involve tedious synthesis procedures and sophisticated assay protocols and require trained personnel for interpretation of responses. On the contrary, our proposed strategy is based on a digital array that is easy-to-use, rapid, and cost-effective. Furthermore, assay results are captured by a digital camera and analyzed using a computer that provides direct readout signals, thereby minimizing human error in interpretation of colorimetric responses. Therefore, the proposed methodology would be ideal for development of point-of-care nucleic acid assay.

\section{CONCLUSIONS}

Although there are reports on fluorescent and colorimetric biosensors using CPE, there is a lack of discussion in the literature on how the sensing capacity of a CPE may vary with structure of the pendant group. Here, by synthesizing PTs and taking advantage of different functional groups in a single polymer, it has been demonstrated that copolymer of PT yielded better optical responses for the miRNA detection in comparison to those of their homopolymers. On the basis of these results, we have developed a novel color-coded array, which is very simplistic in nature for the detection of miRNAs in a complex matrix. Moreover, the proposed assay could be utilized for detection of other RNA sequences of interest upon introduction of a corresponding complementary PNA sequence. Future studies will emphasize on improving $\mathrm{cPT}$ composition and stoichiometric ratios of pendant groups for naked eye detection of miRNA at clinically relevant concentration levels.

\section{ASSOCIATED CONTENT}

\section{S Supporting Information}

The Supporting Information is available free of charge on the ACS Publications website at DOI: 10.1021/acsami.5b12171.

NMR spectra for precursors/monomers and PT, absorbance and emission spectra for PT, delta-E analysis of RGB color intensities, PT emission and absorbance properties, gauss deconvolution of PT spectra, and colorcoded digital array for cPT3.

(PDF)

\section{AUTHOR INFORMATION}

\section{Corresponding Authors}

*E-mail: hakanyildiz@iyte.edu.tr. Phone: +90 2327507527.

*E-mail: bliedberg@ntu.edu.sg. Phone: +65 6316 2957. Fax: $+6567912274$.

\section{Notes}

The authors declare no competing financial interest.

\section{REFERENCES}

(1) Grimsdale, A. C.; Leok Chan, K.; Martin, R. E.; Jokisz, P. G.; Holmes, A. B. Synthesis of Light-Emitting Conjugated Polymers for Applications in Electroluminescent Devices. Chem. Rev. 2009, 109 (3), 897-1091.

(2) Günes, S.; Neugebauer, H.; Sariciftci, N. S. Conjugated PolymerBased Organic Solar Cells. Chem. Rev. 2007, 107 (4), 1324-1338.

(3) Arias, A. C.; MacKenzie, J. D.; McCulloch, I.; Rivnay, J.; Salleo, A. Materials and Applications for Large Area Electronics: Solution-Based Approaches. Chem. Rev. 2010, 110 (1), 3-24.

(4) Thomas, S. W.; Joly, G. D.; Swager, T. M. Chemical Sensors Based on Amplifying Fluorescent Conjugated Polymers. Chem. Rev. 2007, 107 (4), 1339-1386.

(5) Plante, M. P.; Berube, E.; Bissonnette, L.; Bergeron, M. G.; Leclerc, M. Polythiophene Biosensor for Rapid Detection of Microbial Particles in Water. ACS Appl. Mater. Interfaces 2013, 5 (11), 45444548.

(6) Li, C.; Shi, G. Polythiophene-Based Optical Sensors for Small Molecules. ACS Appl. Mater. Interfaces 2013, 5 (11), 4503-4510.

(7) Ma, Y.-Z.; Shaw, R. W.; Yu, X.; O’Neill, H. M.; Hong, K. ExcitedState Dynamics of Water-Soluble Polythiophene Derivatives: Temperature and Side-Chain Length Effects. J. Phys. Chem. B 2012, 116 (49), 14451-14460.

(8) Balamurugan, S. S.; Bantchev, G. B.; Yang, Y.; McCarley, R. L. Highly Water-Soluble Thermally Responsive Poly(thiophene)-Based Brushes. Angew. Chem., Int. Ed. 2005, 44 (31), 4872-4876.

(9) Wang, J.; Zhang, Q.; Tan, K. J.; Long, Y. F.; Ling, J.; Huang, C. Z. Observable Temperature-Dependent Compaction-Decompaction of Cationic Polythiophene in the Presence of Iodide. J. Phys. Chem. B 2011, 115 (7), 1693-1697.

(10) Huser, T.; Yan, M. Solvent-related Conformational Changes and Aggregation of Conjugated Polymers Studied by Single Molecule Fluorescence Spectroscopy. J. Photochem. Photobiol., A 2001, 144 (1), 43-51.

(11) Åsberg, P.; Björk, P.; Höök, F.; Inganäs, O. Hydrogels from a Water-Soluble Zwitterionic Polythiophene: Dynamics under $\mathrm{pH}$ Change and Biomolecular Interactions Observed Using Quartz Crystal Microbalance with Dissipation Monitoring. Langmuir 2005, 21 (16), $7292-7298$.

(12) Harding Lepage, P.; Peytavi, R.; Bergeron, M. G.; Leclerc, M. Amplification Strategy Using Aggregates of Ferrocene-Containing Cationic Polythiophene for Sensitive and Specific Electrochemical Detection of DNA. Anal. Chem. 2011, 83 (21), 8086-8092.

(13) Nilsson, K. P. R.; Inganäs, O. Chip and Solution Detection of DNA Hybridization using a Luminescent Zwitterionic Polythiophene Derivative. Nat. Mater. 2003, 2 (6), 419-424.

(14) Li, C.; Numata, M.; Bae, A.-H.; Sakurai, K.; Shinkai, S. SelfAssembly of Supramolecular Chiral Insulated Molecular Wire. J. Am. Chem. Soc. 2005, 127 (13), 4548-4549.

(15) Zhan, R.; Fang, Z.; Liu, B. Naked-Eye Detection and Quantification of Heparin in Serum with a Cationic Polythiophene. Anal. Chem. 2010, 82 (4), 1326-1333.

(16) Shiraki, T.; Dawn, A.; Tsuchiya, Y.; Yamamoto, T.; Shinkai, S. Unexpected Chiral Induction from Achiral Cationic Polythiophene Aggregates and its Application to the Sugar Pattern Recognition. Chem. Commun. 2012, 48 (56), 7091-7093.

(17) Rubio-Magnieto, J.; Thomas, A.; Richeter, S.; Mehdi, A.; Dubois, P.; Lazzaroni, R.; Clement, S.; Surin, M. Chirality in DNA $-\pi$ Conjugated Polymer Supramolecular Structures: Insights into the SelfAssembly. Chem. Commun. 2013, 49 (48), 5483-5485.

(18) Ho, H.-A.; Najari, A.; Leclerc, M. Optical Detection of DNA and Proteins with Cationic Polythiophenes. Acc. Chem. Res. 2008, 41 (2), $168-178$.

(19) Lee, K.; Povlich, L. K.; Kim, J. Recent Advances in Fluorescent and Colorimetric Conjugated Polymer-based Biosensors. Analyst 2010, 135 (9), 2179-2189. 
(20) Zade, S. S.; Bendikov, M. Twisting of Conjugated Oligomers and Polymers: Case Study of Oligo- and Polythiophene. Chem. - Eur. J. 2007, 13 (13), 3688-3700.

(21) Yildiz, U. H.; Alagappan, P.; Liedberg, B. Naked Eye Detection of Lung Cancer Associated miRNA by Paper Based Biosensing Platform. Anal. Chem. 2013, 85 (2), 820-824.

(22) Zhang, Y.; Li, Z.; Cheng, Y.; Lv, X. Colorimetric Detection of microRNA and RNase $\mathrm{H}$ Activity in Homogeneous Solution with Cationic Polythiophene Derivative. Chem. Commun. 2009, 22, 31723174.

(23) Yao, Z.; Ma, W.; Yang, Y.; Chen, X.; Zhang, Li.; Lin, C.; Wu, H. C. Colorimetric and Fluorescent Detection of Protamines with an Anionic Polythiophene Derivative. Org. Biomol. Chem. 2013, 11 (38), 6466-6469.

(24) Yao, Z.; Hu, X.; Ma, W.; Chen, X.; Zhang, L.; Yu, J.; Zhao, Y.; Wu, H. C. Colorimetric and Fluorescent Dual Detection of Paraquat and Diquat based on an Anionic Polythiophene Derivative. Analyst 2013, 138 (19), 5572-5575.

(25) Lan, M.; Wu, J.; Liu, W.; Zhang, W.; Ge, J.; Zhang, H.; Sun, J.; Zhao, W.; Wang, P. Copolythiophene-Derived Colorimetric and Fluorometric Sensor for Visually Supersensitive Determination of Lipopolysaccharide. J. Am. Chem. Soc. 2012, 134 (15), 6685-6694.

(26) Spangler, C.; Lang, T.; Schferling, M. Spectral Response of a Methylimidazolium-Functionalized Polythiophene to Phosphates. Dyes Pigm. 2012, 95 (2), 194-200.

(27) Zheng, W.; Chase, T. E.; He, L. Multiplexed miRNA Detection using Cationic Polythiophene. Anal. Methods 2014, 6 (7), 2399-2405.

(28) Pu, K. Y.; Liu, B. Intercalating Dye Harnessed Cationic Conjugated Polymer for Real-Time Naked-Eye Recognition of Double-Stranded DNA in Serum. Adv. Funct. Mater. 2009, 19 (9), 1371-1378.

(29) Liu, Z.; Wang, H. L.; Cotlet, M. Energy Transfer from a Cationic Conjugated Polyelectrolyte to a DNA Photonic Wire: Toward Label-Free, Sequence-Specific DNA Sensing. Chem. Mater. 2014, 26 (9), 2900-2906.

(30) Xia, F.; Zuo, X.; Yang, R.; Xiao, Y.; Kang, D.; Vallee-Belisle, A.; Gong, X.; Yuen, J. D.; Hsu, B. B. Y.; Heeger, A. J.; Plaxco, K. W. Colorimetric Detection of DNA, Small Molecules, Proteins, and Ions using Unmodified Gold Nanoparticles and Conjugated Polyelectrolytes. Proc. Natl. Acad. Sci. U. S. A. 2010, 107 (24), 10837-10841.

(31) Fang, B.; Jiao, S.; Li, M.; Qu, Y.; Jiang, X. Label-Free Electrochemical Detection of DNA using Ferrocene-containing Cationic Polythiophene and PNA Probes on Nanogold Modified Electrodes. Biosens. Bioelectron. 2008, 23 (7), 1175-1179. 